\title{
The Role of Ischemia-Reperfusion Injury in Reconstructive Transplantation
}

\section{Saami Khalifian, Damon S Cooney, WP Andrew Lee and Gerald Brandacher*}

Department of Plastic and Reconstructive Surgery, Vascularized Composite Allotransplantation (VCA) Laboratory, Johns Hopkins University School of Medicine, Baltimore, MD, USA

\begin{abstract}
Reconstructive transplantation, also referred to as vascularized composite allotransplantation (VCA), has rapidly emerged as a viable approach to repairing complex tissue defects. Over the past 20 years, major advances have been made in the field of VCA allowing for successful transplantation of over 150 hand/forearm/arm, larynx, trachea, abdominal wall, vascularized knee, and facial transplants with encouraging outcomes. These innovations have currently outpaced the scientific community's ability to fully address certain immunological and clinical challenges. The literature on ischemia-reperfusion injury (IRI) in VCA is limited and mechanistic questions remain. Specifically, the role IRI may play in acute rejection, the progression towards chronic rejection, or immune regulation and tolerance induction has only been partially or indirectly addressed. Hence, much of what we understand regarding IRI in VCA is extrapolated from research in solid organ transplantation (SOT). This review will address the role of IRI in VCA, first outlining its impact on SOT, it's effects on the immune system and allograft rejection, as well as the clinical implications that IRI has for VCA outcomes.
\end{abstract}

Keywords: Ischemia-Reperfusion Injury; Vascularized composite allotransplantation; Transplant; Immunity; Rejection

\section{IRI and Organ Transplantation}

The literature in SOT has clearly demonstrated that IRI is a potent activator of the immune system and subsequently leads to poor functional outcomes [1-4]. IRI poses a unique challenge for the transplant surgeon, as some period of ischemia is unavoidable given the nature of the procedure. Ischemia, induced by donor organ procurement, cold preservation, and implantation, is one of several risk factors shown to contribute to both acute and chronic rejection [5]. Reperfusion injury further compromises the viability of transplanted tissues as the restoration of blood flow leads to activation of the complement system, generation of reactive oxygen species (ROS), and the production of pro-inflammatory cytokines that intensify damage in the previously ischemic graft [6]. It has been shown that IRI leads to increased expression of MHC antigens and activates the innate immune system producing inflammation, ultimately leading to acute rejection, hastens the development of chronic rejection, and impaired chances for immune regulation and tolerance induction [5].

In cardiac transplantation, IRI is common in the early posttransplant period, especially when ischemic time exceeds two hours, and is characterized by hyperemia in the previously ischemic myocardium, which later becomes prone to coagulative necrosis [7]. Studies have shown that prolonged ischemia in transplanted hearts leads to increased entry of activated $\mathrm{T}$ cells and binding of natural IgM antibodies to self-antigens exposed after tissue ischemia $[8,9]$. The former leads to leukocyte migration and accumulation in peripheral tissues and the latter is a potent activator of the complement cascade. Together, these effects are major contributors to the acute rejection and late graft loss seen after IRI [10]. Indeed, patients with cardiac allografts subjected to increased ischemic times are at increased risk for early graft loss, early death after transplant, and coronary artery vasculopathy [11]. Furthermore, prolonged ischemia was found to be an independent risk factor for mortality at 1 and 10 years post-transplant [11].

Similarly, animal models of renal transplants have shown that prolonged ischemia time leads to more acute rejection episodes [12]. Subsequent clinical trials observed the same effect on human renal transplants. Analysis of over 6,000 kidney transplant recipients showed that patients with prolonged ischemic times suffered early acute rejection and decreased 6-year renal graft survival [13]. These findings were independent of donor/recipient age, HLA mismatch, reactive antibodies, and early rejection treatments. Notably, acute transplant rejections were found to be a significant risk factor for short and longterm graft survival [14]. Indeed, IRI predisposes all types of transplanted grafts to unfavorable sequelae. In lung and liver allografts, IRI leads to higher incidences of acute and chronic rejection $[5,15]$. However, there is a paucity of studies examining IRI in vascularized composite allotransplantation. It is possible that vascularized composite allografts may be even more susceptible to IRI given the diversity of tissue components contained within the graft [16].

\section{Molecular Foundations and Markers of IRI}

IRI mediates tissue injury by producing a strong inflammatory response that activates the immune system. This immune activation is mediated by upregulation of inflammatory cytokines and adhesion molecules, activation of leukocytes and endothelial cells, and generation of oxygen free radicals [17]. The duration of ischemia corresponds to the level of immune activation, with increased ischemic times leading to increased expression of inflammatory cytokines, such as TNF- $\alpha$, IFN- $\gamma$, and IL- $1[17,18]$. Interaction of cell adhesion molecules on activated leukocytes with their ligands on the injured endothelium leads to diapedesis of these cells into the interstitial space. These activated leukocytes damage tissues through the release of proteolytic enzymes and the generation of ROS that propagate the injury response. Ischemia produces further tissue damage as elevated glycolysis causes lactic acid accumulation, $\mathrm{pH}$ reduction, and impairment of membrane

*Corresponding author: Gerald Brandacher, Scientific Director, Reconstructive Transplantation Program, Department of Plastic and Reconstructive Surgery, Johns Hopkins University School of Medicine, Ross Research Building 749D, 720 Rutland Avenue, Baltimore, MD21205,USA.Tel: (443) 287-6679; Fax: (410) 614-1296; E-mail: brandacher@jhmi.edu

Received May 03, 2013; Accepted June 07, 2013; Published June 10, 2013

Citation: Khalifian S, Cooney DS, Andrew Lee WP, Brandacher G (2013) The Role of Ischemia-Reperfusion Injury in Reconstructive Transplantation. J Transplant Technol Res S3: 004. doi:10.4172/2161-0991.S3-004

Copyright: (c 2013 Khalifian S, et al. This is an open-access article distributed under the terms of the Creative Commons Attribution License, which permits unrestricted use, distribution, and reproduction in any medium, provided the original author and source are credited. 
transport functions [19]. The ischemic tissue will undergo necrosis unless perfusion is restored. Paradoxically, reperfusion leads to injury beyond that caused by the ischemia through the production of ROS in the mitochondria [20].

The resulting tissue injury leads to the generation of damageassociated molecular patterns (DAMPs), which are recognized by pattern-recognition receptors (PRRs) of the innate immune system [21]. Activation of PRRs leads to upregulation of inflammatory cytokines and adhesion molecules, which contribute to recruitment of leukocytes into the graft and to transplant vasculopathy [21,22]. ROS further trigger the innate immune system via activation of heat shock proteins (HSP) that signal toll-like receptors (TLRs) on macrophages and B cells [23]. However, TLRs play a dual immunological role by also activating antigen-presenting cells (APCs)- the gatekeepers of the adaptive immune system. The sensitization of APCs by TLRs leads to a dramatic increase of effector T-cells $[22,24,25]$. This alloreactive $\mathrm{T}$ cell response is further augmented by the pro-inflammatory cytokine milieu induced by IRI [25]. Thus, ROS activate both the innate and adaptive immune system, therefore contributing to trigger both acute and chronic rejection. It is important to note, however, that the link between non-specific injury, such as IRI, and the innate immune system is still speculative [26]. Although there is biological plausibility, unequivocal evidence of this correlation does not yet exist.

Another key event in IRI is activation of the alternative complement pathway. Complement is considered to be a key component leading to rejection of transplanted organs by damaging cell membranes through the formation of membrane attack complexes, and attracting neutrophils to sites of IRI via chemotactic agents such as C5a $[6,10,17,19,27]$. Complement also releases anaphylatoxins (C3a, C5a) that cause degranulation of mast cell and the release of histamine, which further damages tissues.

\section{IRI, Rejection and Tolerance}

The most important factor leading to transplant rejection is undoubtedly the $\mathrm{T}$ cell response triggered by MHCincompatibility [28]. This response is further bolstered by IRI, which is believed to be the strongest secondary factor to increase graft allogenicity [12]. Ischemic injury predisposes to immunologic recognition and rejection through upregulation of MHC II (signal 1) and costimulation (signal 2) by activation of APCs [4]. There is ample evidence that multiple episodes of acute rejection initiate a state of chronic inflammation that may trigger myointimal proliferation, allograft vessel occlusion, and chronic graft dysfunction [4,5,15,29-31]. For example, using a rat hindlimb model of VCA, Unadkat et al. showed that acute rejection episodes lead to composite tissue vasculopathy and degeneration [32]. Thus, IRI induces an immune response, which leads to further injury, promoting a new injury response and increased immune recognition. This self-perpetuating feature may lead to chronic rejection and irreversible tissue damage [4].

Large clinical trials in SOT have demonstrated a clear relationship between the duration of ischemia and graft survival [33]. It is hypothesized that this link is mediated by TLRs that are activated by ROS generated by IRI [5]. TLRs have been shown to be key contributors to the rate of acute rejection in skin [34] heart [35], lung [5], kidney [36], liver [3], and islet [37] allografts. In a fully MHC-mismatched model of skin allografts, MyD88 (a TLR signal adaptor) knockout mice accepted their allografts indefinitely after administration of costimulatory blockade (CTLA4-Ig and anti-CD154), while wild-type animals went on to reject the allograft [38]. Systemic administration of a TLR activator $(\mathrm{CpG})$ eliminated transplant tolerance induction in skin allografts [39], which together strongly suggests that TLR signaling impairs transplant tolerance [40]. Similarly, in a fully MHCmismatched model of kidney allografts, absence of a negative regulator of TLRs enhanced IRI and induced DC maturation with a more robust acute rejection response [41]. The absence of this negative regulator also impaired transplant tolerance induction and enhanced the development of chronic rejection in the same study. In humans, kidney allograft recipients with non-responsiveness to their allografts showed lower expression of MyD88 than recipients with chronic rejection [42]. TLR gene expression was also associated with endothelial dysfunction and vasculopathy in clinical studies of cardiac transplant [22]. Furthermore, GWAS studies have shown that hyporesponsive TLR genes are associated with improved outcomes for transplant recipients exemplified by fewer rejection episodes and improved graft function $[1,2,43]$. Although translational research studying VCA rejection is limited, it is likely that the activation of TLRs by IRI also contributes to the acute rejection episodes that occur in $85 \%$ of hand transplant recipients in the first year [30]. By dampening the effects of IRI, it may be possible to mitigate the activation of the immune system that leads to acute and chronic rejection.

\section{Animal Studies of IRI}

There are a limited number of mechanistic studies exploring the effects of IRI in VCA. It is likely that IRI is more pronounced in VCA than it is in SOT due to the heterogeneity of the graft together with the highly antigenic skin component [16]. However, studies have shown that the most vulnerable site of injury in IRI is actually the muscle due to its high metabolic activity [19]. In as little as 3 hours of ischemia, a significant increase in the early signs of acute rejection can be observed in the skin and muscle. With 3 hours of ischemia, early signs of irreversible skeletal myocyte damage appear and this process is usually complete by 6 hours [44]. In a rat vascularized epigastric allotransplant model, an increase inischemia from 1 hour to 3 hours led to more acute rejection episodes, a more severe inflammatory response, and higher levels of activated lymphocytes [30]. This relatively short period of ischemia, which is within the period of "reversible" damage, is enough to tip the balance towards chronic rejection of the composite tissue allograft. A more dramatic difference in ischemia time (1 hour vs. 6 hours) in an allo-skin flap model caused an even more severe and rapid onset of rejection [17]. In vitro assays showed that grafts with less ischemia time exhibited lower anti-donor lymphocyte proliferation in mixed lymphocyte reactions and lower MHC-II expression. This inflammatory response leads to dysfunction of endothelial cells, triggering a reduction in nitric oxide (NO) release [19]. Consequentially, the tissue-protective effects of NO are minimized, such as attenuation of leukocyte adherence to the endothelium, scavenging of ROS, and physiologic regulation of vascular tone [45]. Microvascular insufficiency and inflammation in postcapillary venules ensues giving rise to the "no-reflow phenomenon" and ultimately, acute rejection of the composite tissue allograft [46]. Furthermore, prolonged ischemia was shown to have deleterious effects in a rat groin flap, with an increased proportion of CXCR3-positive cells in the graft, which may play a role in the rejection process [47].

As in SOT, reperfusion causes more damage than ischemia [48]. In a rat soleus muscle allograft, histologic damage was proportionate to reperfusion time [49]. Increased reperfusion also amplifies the rate of ROS formation in limbs after revascularization, and impairs vasodilation of the feeding arteries [50]. Steps can be taken to reduce the sequelae of IRI such as local hypothermia $\left(10^{\circ} \mathrm{C}\right.$ versus $\left.34^{\circ} \mathrm{C}\right)$, which has been shown to significantly reduce microvascular permeability [51]. More mechanistic studies of IRI in VCA may help elucidate how we can shift 
the balance of immune regulation after IRI from immunostimulation to immunomodulation.

\section{Clinical Outcomes of IRI in VCA}

Although it is clear that IRI portends poor outcomes in reconstructive transplantation, there is no available clinical data examining the relationship between ischemia time and acute and chronic rejection in VCA. As a result, inferences must be made based on the available data from SOT as well as the animal studies described above. As more experience has been gained in the field of VCA, ischemia times have decreased for these complex operations. Ischemia time for hand and face transplant now average between 6 and 4 hours, respectively [31]; however, irreversible skeletal myocyte damage begins after only 3 hours of ischemia [44]. This amount of prolonged ischemia time may account for differences in composite allograft dysfunction, such as that seen between two bilateral hand transplant patients (160 minutes versus 9 hours) [52]. IRI may also be a key determinant of acute rejection in $85 \%$ of hand transplants in the first year, as prolonged ischemia intensifies the rate and severity of acute rejection. This may be demonstrated by a 30 -year-old patient with a right hand and left hand-forearm double allograft. The more complex hand-forearm dissection led to perioperative ischemic injury of the forearm allograft. Consequentially, the patient developed flexion contractures of the forearm and the graft subsequently suffered three rejection episodes [7]. While it is impossible to unequivocally determine whether the cause of this patient's rejection episodes were due to prolonged ischemia time, this case does lend credence to the notion that increased ischemia portends poor outcomes in VCA. As more clinical data becomes available in VCA, it will be important for this issue to be analyzed to clearly demonstrate the association between prolonged ischemia with severity and frequency of rejection episodes.

Given the profound effect IRI can have on transplanted tissues, it is important to gain insight into the status of the transplanted graft prior to the onset of clinical rejection. Close monitoring of composite tissue allografts will help combat the acute rejection episodes that increase the risk of chronic graft dysfunction. Typically, the skin is the principal target of rejection after VCA, as it is the most immunogenic component of the graft $[16,53]$. Due to its external location, the skin provides a unique clinical opportunity for the monitoring, early diagnosis, prevention and treatment of VCA rejection. If ischemia times are prolonged then the risk of acute rejection is higher and the markers of rejection will appear more rapidly $[6,17,30]$. A study examining 170 biopsies from 5 hand transplant patients revealed upregulation of adhesion molecules during skin rejection with the severity of rejection strongly correlated with LFA-1, ICAM-1, and E-selectin [54]. However, skin and fascia are more resistant to IRI than muscle [27]. This is due to a more active metabolism in muscle as well as significant differences in reflow patterns between the tissues after IRI [19]. Histologic signs of IRI usually peak around 24-48 hours after the onset of ischemia, whereas clinical signs of acute rejection appear days later [30]. Clinical markers of IRI include wet-to-dry-weight ratio, nitroblue tetrazolium, tissue lactate, tissue myeloperoxidase, and laser Doppler flow [31]. Of these, nitroblue tetrazolium \& wet-to-dry-weight ratio are considered to be the most accurate predictors of outcome after IRI in VCA [30]. Creatine kinase [CK] and aspartate transaminase [AST] levels also demonstrate the effects of prolonged ischemia on skeletal muscle. Using a gracilis myocutaneous flap, Villamaria et al. demonstrated significantly elevated CK and AST levels after a 1 vs. 3-hour delay in restoration of blood flow [6]. Given that skeletal muscle is the most sensitive tissue to IRI, monitoring of CK and AST levels may also be appropriate. These markers provide important data regarding acute rejection episodes that guide clinical management and immunosuppression drug levels.

Further insights may be gleaned from data on replantation of digits or whole limbs. In one series, the mean survival for digit replantation after complete avulsion was $66 \%(n=442)$, but lower for limb replantation due to IRI of skeletal muscle [55]. Replantation and revascularization of ischemic whole limbs causes acidosis, hyperkalemia, myoglobinuria, and can lead to hypotension from obstruction of the renal vasculature [27]. In the setting of limb replantation, ROS and cytokines (such as TNF $\alpha$ ) can lead to capillary leakage and pulmonary edema [56]. Compartment syndrome is another dreaded complication after limb revascularization and can lead to muscle necrosis, interstitial fibrosis, and nerve scarring [57]. The combination of these factors often necessitates re-amputation of the newly re-attached limb. Clearly, the challenges faced by the patient early after transplantation are numerous as many of the aforementioned sequelae are possible in patients after VCA [20]. Thus, it is important to minimize ischemia time and the effects of IRI to give the patient the best chance of a successful recovery.

\section{Prevention and Treatment of IRI}

While it is currently impossible to perform VCA with no ischemia time, every effort should be made to minimize the duration. Prevention of IRI may be achieved via application of therapies that have shown promise in SOT, such as pre-conditioning, hypothermia, preservation solutions, and antioxidants. Ischemic preconditioning is one of the most popular methods of preventing IRI. By inducing brief episodes of ischemia interrupted by intermittent reperfusion, the graft becomes more resistant to IRI $[58,59]$. The protective effect of preconditioning is related to duration and number of each ischemia-reperfusion cycle. There is evidence that longer (10 min. versus $5 \mathrm{~min}$.) and more frequent cycles ( 3 cycles versus 1 or 2 cycles) are superior at reducing the extent of IRI [60]. Nitric oxide appears to be a principal protective factor. Ischemic preconditioning upregulates numerous isoforms of nitric oxide synthase and the protective effect is lost by administration of a nonselective nitric oxide synthase inhibitor [61]. Numerous studies have demonstrated prolonged graft survival and improved outcomes from pre-conditioning $[19,20,27,31]$. For example, the use of preconditioning and the antioxidant resveratrol in animal VCA models was shown to be protective against ischemia-induced microcirculatory changes and acute rejection [62]. Other studies showed that preconditioning led to reduced leukocyte-endothelial interactions and apoptosis, while improving microvascular perfusion in a rat hindlimb model [63]. Transient limb ischemia can also remotely confer the beneficial effects of pre-conditioning to other areas [64]. By inducing transient ischemia in the non-transplanted limb, one can dampen IRI in the transplanted limb or other graft [20]. In mouse [64], rat [65], and swine [66] models, it was shown that remote ischemic pre-conditioning provided local and remote protection against IRI, improved survival, and reduced infarct size.

By manipulating the cytokine milieu pre-injury, we may prevent overstimulation of the inflammatory response that leads to acute rejection. One approach is through the induction of local hypothermia [67]. This reduces leukocyte adhesion and infiltration into tissues and minimizes the generation of ROS. Furthermore, hypothermia has been shown to prolong graft viability and enhance survival of replanted limbs $[68,69]$. However, skeletal muscle is susceptible to cold IRI as well [70]. This can be addressed through the use of preservation solutions, such as University of Wisconsin (UW) or Histidine-tryptophan-ketoglutarate (HTK) solution. Intra-arterial flushing of limbs with cold UW or HTK solution causes uniform temperature reduction that can diminish cold 
IRI in skeletal muscle [71]. Pulsatile perfusion provides nutrients to the graft and flushes out harmful toxins [72]. It also prevents expression of proinflammatory cytokines and helps maintain capillary perfusion. Hypothermia and pulsatile perfusion with UW solution are currently standard practice in VCA, however, the advent of new preservation solutions such as polyethylene glycol and Celsior solution, amongst others, may prove to be even more efficacious in minimizing IRI.

Another promising strategy to eliminate IRI is to quell the ROS that triggers reperfusion injury. Antioxidants serve this purpose and have been shown to improve outcomes after transplant by reducing histological damage and improving mitochondrial dysfunction [20]. A variety of antioxidants have been investigated including superoxide dismutase, Tempol, Coenzyme Q10, zinc aspartate, taurine and others. Heme oxygenase 1 showed promising results in kidney transplant patients by demonstrating improved graft function with no effect on immunosuppression drug levels [73,74]. However, no single antioxidant or combination has demonstrated consistent reduction of IRI in randomized controlled trials.

Therapeutic interventions should also be utilized post-transplant. Biopsies 24-48 hours after transplant will provide insight into the health and immune status of the graft. Since ischemically-challenged skeletal muscle is more prone to acute rejection, muscle tissue should not be excluded from surveillance biopsies [30]. Evidence of myositis on biopsy may be a harbinger of rejection, as was seen in two VCA patients whose grafts were beginning to reject [75]. Similarly, adipose and subcutaneous tissue should also be included, because these tissues all provide valuable information regarding early acute rejection.

\section{Future Directions of IRI research in VCA}

Some amount of ischemia is unavoidable in VCA; however, the consequences of IRI may potentially be mitigated. Further studies elucidating the role of IRI in activating the innate and adaptive immune response may clearly define the targets that must be modulated in order to prevent tissue damage or allograft rejection. It is likely that there is a unique, differential release of innate immune ligands after IRI, but this is an under-investigated area [5]. Major insights may be gained into IRI by correlating duration of ischemia-reperfusion with the following: characterization of cytokine expression, upregulation of MHC antigens, and identification of self-antigens that activate IgM and complement. These findings could help direct the development of new therapies. Clinically, studies are needed in order to characterize the short and long-term risks associated with oxidative damage. To address the issue of rejection and graft surveillance, studies should evaluate biopsies taken in the 24-48 hour window after transplantation to detect IRI before signs of acute rejection appear [30]. Developing a clinical measure of IRI at an earlier time point will allow for interventions prior to the development of an unfavorable cytokine milieu. Ideally, a dose response could be developed that would correlate duration of ischemia with histological findings from early biopsies, thus providing the surgical team with an early window for interventions. Continued research in the development and evaluation of therapies that diminish IRI will help facilitate the minimization of immunosuppression or induction of tolerance after VCA.

"Variability is the law of life, and as no two faces are the same, so no two bodies are alike, and no two individuals react alike and behave alike under the abnormal conditions which we know as disease" -- William Osler

\section{References}

1. Palmer SM, Burch LH, Davis RD, Herczyk WF, Howell DN, et al. (2003) The role of innate immunity in acute allograft rejection after lung transplantation. Am J Respir Crit Care Med 168: 628-632.

2. Krüger B, Krick S, Dhillon N, Lerner SM, Ames S, et al. (2009) Donor Tolllike receptor 4 contributes to ischemia and reperfusion injury following human kidney transplantation. Proc Natl Acad Sci U S A 106: 3390-3395.

3. Zhai Y, Shen XD, O'Connell R, Gao F, Lassman C, et al. (2004) Cutting edge: TLR4 activation mediates liver ischemia/reperfusion inflammatory response via IFN regulatory factor 3-dependent MyD88-independent pathway. J Immuno 173: $7115-7119$

4. Halloran PF, Homik J, Goes N, Lui SL, Urmson J, et al. (1997) The "injury response": a concept linking nonspecific injury, acute rejection, and long-term transplant outcomes. Transplant Proc 29: 79-81.

5. Kreisel D, Goldstein DR (2013) Innate immunity and organ transplantation: focus on lung transplantation. Transpl Int 26: 2-10.

6. Villamaria CY, Rasmussen TE, Spencer JR, Patel S, Davis MR (2012) Microvascular porcine model for the optimization of vascularized composite tissue transplantation. J Surg Res 178: 452-459.

7. Landin L, Cavadas PC, Garcia-Cosmes P, Thione A, Vera-Sempere F (2011) Perioperative ischemic injury and fibrotic degeneration of muscle in a forearm allograft: functional follow-up at 32 months post transplantation. Ann Plast Surg 66: 202-209.

8. Chalasani G, Li Q, Konieczny BT, Smith-Diggs L, Wrobel B, et al. (2004) The allograft defines the type of rejection (acute versus chronic) in the face of an established effector immune response. J Immunol 172: 7813-7820.

9. Zhang M, Carroll MC (2007) Natural antibody mediated innate autoimmune response. Mol Immunol 44: 103-110.

10. Reichenbach DK, Li Q, Hoffman RA, Williams AL, Shlomchik WD, et al. (2013) Allograft outcomes in outbred mice. Am J Transplant 13: 580-588.

11. Boucek MM, Aurora P, Edwards LB, Taylor DO, Trulock EP, et al. (2007) Registry of the International Society for Heart and Lung Transplantation: tenth official pediatric heart transplantation report--2007. J Heart Lung Transplant 26 796-807.

12. Tullius SG, Reutzel-Selke A, Egermann F, Nieminen-Kelhä M, Jonas S, et al. (2000) Contribution of prolonged ischemia and donor age to chronic renal allograft dysfunction. J Am Soc Nephrol 11: 1317-1324

13. Salahudeen AK, Haider N, May W (2004) Cold ischemia and the reduced long term survival of cadaveric renal allografts. Kidney Int 65: 713-718.

14. Ojo AO, Wolfe RA, Held PJ, Port FK, Schmouder RL (1997) Delayed graft function: risk factors and implications for renal allograft survival. Transplantation 63: 968-974

15. Hautz T, Wolfram D, Grahammer J, Starzl R, Krapf C, et al. (2012) Mechanisms and mediators of inflammation: potential models for skin rejection and targeted therapy in vascularized composite allotransplantation. Clin Dev Immunol 2012:757310.

16. Lee WP, Yaremchuk MJ, Pan YC, Randolph MA, Tan CM, et al. (1991) Relative antigenicity of components of a vascularized limb allograft. Plast Reconstr Surg 87: 401-411.

17. Shimizu F, Okamoto O, Katagiri K, Fujiwara S, Wei FC (2010) Prolonged ischemia increases severity of rejection in skin flap allotransplantation in rats. Microsurgery 30: 132-137.

18. Kuo YR, Wang FS, Jeng SF, Huang HC, Wei FC, et al. (2004) Nitrosoglutathione modulation of platelet activation and nitric oxide synthase expression in promotion of flap survival after ischemia/reperfusion injury. J Surg Res 119 92-99

19. Siemionow M, Arslan E (2004) Ischemia/reperfusion injury: a review in relation to free tissue transfers. Microsurgery 24: 468-475.

20. Wang WZ, Baynosa RC, Zamboni WA (2011) Update on ischemia-reperfusion injury for the plastic surgeon: 2011. Plast Reconstr Surg 128: 685e-92e.

21. Akira S, Uematsu S, Takeuchi O (2006) Pathogen recognition and innate immunity. Cell 124: 783-801.

22. Methe H, Zimmer E, Grimm C, Nabauer M, Koglin J (2004) Evidence for a role of toll-like receptor 4 in development of chronic allograft rejection after cardiac transplantation. Transplantation 78: 1324-1331.

23. Akira S, Takeda K, Kaisho T (2001) Toll-like receptors: critical proteins linking innate and acquired immunity. Nat Immunol 2: 675-680. 
Citation: Khalifian S, Cooney DS, Andrew Lee WP, Brandacher G (2013) The Role of Ischemia-Reperfusion Injury in Reconstructive Transplantation. J Transplant Technol Res S3: 004. doi:10.4172/2161-0991.S3-004

24. Gill RG (2010) NK cells: elusive participants in transplantation immunity and tolerance. Curr Opin Immunol 22: 649-654.

25. Ionita MG, Arslan F, de Kleijn DP, Pasterkamp G (2010) Endogenous inflammatory molecules engage Toll-like receptors in cardiovascular disease. J Innate Immun 2: 307-315.

26. Matzinger P (1998) An innate sense of danger. Semin Immunol 10: 399-415

27. Khalil AA, Aziz FA, Hall JC (2006) Reperfusion injury. Plast Reconstr Surg 117: 1024-1033.

28. Morelli AE, Thomson AW (2007) Tolerogenic dendritic cells and the quest for transplant tolerance. Nat Rev Immunol 7: 610-621.

29. Kaufman CL, Ouseph R, Blair B, Kutz JE, Tsai TM, et al. (2012) Graft vasculopathy in clinical hand transplantation. Am J Transplant 12: 1004-1016.

30. Pradka SP, Ong YS, Zhang Y, Davis SJ, Baccarani A, et al. (2009) Increased signs of acute rejection with ischemic time in a rat musculocutaneous allotransplant model. Transplant Proc 41: 531-536.

31. Caterson EJ, Lopez J, Medina M, Pomahac B, Tullius SG (2013) Ischemiareperfusion injury in vascularized composite allotransplantation. J Craniofac Surg 24: 51-56.

32. Unadkat JV, Schneeberger S, Horibe EH, Goldbach C, Solari MG, et al (2010) Composite tissue vasculopathy and degeneration following multiple episodes of acute rejection in reconstructive transplantation. American journal of transplantation 10: 251-261.

33. Opelz G, Wujciak T (1994) The influence of HLA compatibility on graft surviva after heart transplantation. The Collaborative Transplant Study. N Engl J Med 330: 816-819.

34. McKay D, Shigeoka A, Rubinstein M, Surh C, Sprent J (2006) Simultaneous deletion of MyD88 and Trif delays major histocompatibility and minor antigen mismatch allograft rejection. Eur J Immunol 36: 1994-2002.

35. Tesar BM, Zhang J, Li Q, Goldstein DR (2004) TH1 immune responses to fully $\mathrm{MHC}$ mismatched allografts are diminished in the absence of MyD88, a toll-like receptor signal adaptor protein. Am J Transplant 4: 1429-1439.

36. Wu H, Chen G, Wyburn KR, Yin J, Bertolino P, et al. (2007) TLR4 activation mediates kidney ischemia/reperfusion injury. J Clin Invest 117: 2847-2859.

37. Hutton MJ, Westwell-Roper C, Soukhatcheva G, Plesner A, Dutz JP, et al (2009) Islet allograft rejection is independent of toll-like receptor signaling in mice. Transplantation 88: 1075-1080.

38. Walker WE, Nasr IW, Camirand G, Tesar BM, Booth CJ, et al. (2006) Absence of innate MyD88 signaling promotes inducible allograft acceptance. J Immunol 177: 5307-5316.

39. Thornley TB, Brehm MA, Markees TG, Shultz LD, Mordes JP, et al. (2006) TLR agonists abrogate costimulation blockade-induced prolongation of skin allografts. J Immunol 176: 1561-1570.

40. Chen L, Wang T, Zhou P, Ma L, Yin D, et al. (2006) TLR engagement prevents transplantation tolerance. Am J Transplant 6: 2282-2291.

41. Noris M, Cassis P, Azzollini N, Cavinato R, Cugini D, et al. (2009) The TollIL-1R member Tir8/SIGIRR negatively regulates adaptive immunity against kidney grafts. J Immunol 183: 4249-4260.

42. Braudeau C, Ashton-Chess J, Giral M, Dugast E, Louis S, et al.(2008) Contrasted blood and intragraft toll-like receptor 4 mRNA profiles in operational tolerance versus chronic rejection in kidney transplant recipients. Transplantation 86: $130-136$.

43. Ducloux D, Deschamps M, Yannaraki M, Ferrand C, Bamoulid J, et al. (2005) Relevance of Toll-like receptor-4 polymorphisms in renal transplantation. Kidney Int 67: 2454-2461.

44. Blaisdell FW (2002) The pathophysiology of skeletal muscle ischemia and the reperfusion syndrome: a review. Cardiovasc Surg 10: 620-630.

45. Lefer AM, Lefer DJ (1996) The role of nitric oxide and cell adhesion molecules on the microcirculation in ischaemia-reperfusion. Cardiovasc Res 32: 743-751.

46. Scott Isenberg J (2003) Modulating effects of L-arginine on cytokine-stimulated lymphocyte migration in vitro. Microsurgery 23: 262-267.

47. Xiao B, Xia W, Zhang J, Liu B, Guo S (2010) Prolonged cold ischemic time results in increased acute rejection in a rat allotransplantation model. J Surg Res 164: e299-304.
48. Loerakker S, Oomens CW, Manders E, Schakel T, Bader DL, et al. (2011) Ischemia-reperfusion injury in rat skeletal muscle assessed with T2-weighted and dynamic contrast-enhanced MRI. Magn Reson Med 66: 528-537.

49. Carmo-Araújo EM, Dal-Pai-Silva M, Dal-Pai V, Cecchini R, Anjos Ferreira $\mathrm{AL}$ (2007) Ischaemia and reperfusion effects on skeletal muscle tissue: morphological and histochemical studies. Int J Exp Pathol 88: 147-154.

50. Arató E, Jancsó G, Sínay L, Kürthy M, Lantos J, et al. (2008) Reperfusion injury and inflammatory responses following acute lower limb revascularization surgery. Clin Hemorheol Microcirc 39: 79-85.

51. Ward R, Souder N, Stahl D, Hunter F, Probe R, et al. (2009) The role of nitric oxide synthase and heme oxygenase in the protective effect of hypothermia in ischemia-reperfusion injury. J Bone Joint Surg Am 91: 2637-2645

52. Herzberg G, Weppe F, Masson N, Gueffier X, Erhard L (2008) Clinica evaluation of two bilateral hand allotransplantations at six and three years follow-up. Chir Main 27: 109-117.

53. Swearingen B, Ravindra K, Xu H, Wu S, Breidenbach WC, et al. (2008) Science of composite tissue allotransplantation. Transplantation 86: 627-635.

54. Hautz T, Zelger B, Grahammer J, Krapf C, Amberger A, et al. (2010) Molecular markers and targeted therapy of skin rejection in composite tissue allotransplantation. Am J Transplant 10: 1200-1209.

55. Davis Sears E, Chung KC (2011) Replantation of finger avulsion injuries: a systematic review of survival and functional outcomes. J Hand Surg Am 36: 686-694.

56. Gilmont RR, Dardano A, Engle JS, Adamson BS, Welsh MJ, et al. (1996) TNFalpha potentiates oxidant and reperfusion-induced endothelial cell injury. J Surg Res 61: 175-182.

57. Kim JY, Schierle CF, Subramanian VS, Birman MV, Kloeters O, et al. (2009) A prognostic model for the risk of development of upper extremity compartment syndrome in the setting of brachial artery injury. Ann Plast Surg 62: 22-27.

58. Yin DP, Sankary HN, Chong AS, Ma LL, Shen J, et al. (1998) Protective effect of ischemic preconditioning on liver preservation-reperfusion injury in rats. Transplantation 66: 152-157.

59. Mounsey RA, Pang CY, Forrest C (1992) Preconditioning: a new technique for improved muscle flap survival. Otolaryngol Head Neck Surg 107: 549-552.

60. Zahir TM, Zahir KS, Syed SA, Restifo RJ, Thomson JG (1998) Ischemic preconditioning of musculocutaneous flaps: effects of ischemia cycle length and number of cycles. Ann Plast Surg 40: 430-435.

61. Huang SS, Wei FC, Hung LM (2006) Ischemic preconditioning attenuates postischemic leukocyte--endothelial cell interactions: role of nitric oxide and protein kinase C. Circ J 70: 1070-1075.

62. Hsieh YH, Huang SS, Wei FC, Hung LM (2007) Resveratrol attenuates ischemia - reperfusion-induced leukocyte - endothelial cell adhesive interactions and prolongs allograft survival across the MHC barrier. Circ J 71: 423-428.

63. Schoen M, Rotter R, Gierer P, Gradl G, Strauss U, et al. (2007) Ischemic preconditioning prevents skeletal muscle tissue injury, but not nerve lesion upon tourniquet-induced ischemia. J Trauma 63: 788-797.

64. Eberlin KR, McCormack MC, Nguyen JT, Tatlidede HS, Randolph MA, et al. (2008) Ischemic preconditioning of skeletal muscle mitigates remote injury and mortality. J Surg Res 148: 24-30.

65. Zahir KS, Syed SA, Zink JR, Restifo RJ, Thomson JG (1998) Ischemic preconditioning improves the survival of skin and myocutaneous flaps in a rat model. Plast Reconstr Surg 102: 140-150.

66. Harkin DW, Barros D'Sa AA, McCallion K, Hoper M, Campbell FC (2002) Ischemic preconditioning before lower limb ischemia--reperfusion protects against acute lung injury. J Vasc Surg 35: 1264-1273.

67. Mowlavi A, Neumeister MW, Wilhelmi BJ, Song YH, Suchy H, et al. (2003) Local hypothermia during early reperfusion protects skeletal muscle from ischemia-reperfusion injury. Plast Reconstr Surg 111: 242-250.

68. Sapega AA, Heppenstall RB, Sokolow DP, Graham TJ, Maris JM, et al. (1988) The bioenergetics of preservation of limbs before replantation. The rationale for intermediate hypothermia. J Bone Joint Surg Am 70: 1500-1513.

69. Usui M, Ishii S, Muramatsu I, Takahata N (1978) An experimental study on "replantation toxemia". The effect of hypothermia on an amputated limb. J Hand Surg Am 3: 589-596. 
Citation: Khalifian S, Cooney DS, Andrew Lee WP, Brandacher G (2013) The Role of Ischemia-Reperfusion Injury in Reconstructive Transplantation. J Transplant Technol Res S3: 004. doi:10.4172/2161-0991.S3-004

70. Wagh M, Pantazi G, Romeo R, Hurley JV, Morrison WA, et al. (2000) Cold storage of rat skeletal muscle free flaps and pre-ischemic perfusion with modified UW solution. Microsurgery 20: 343-349.

71. Rosen HM, Slivjak MJ, McBrearty FX (1987) The role of perfusion washout in limb revascularization procedures. Plast Reconstr Surg 80: 595-605.

72. Yuan X, Theruvath AJ, Ge X, Floerchinger B, Jurisch A, et al. (2010) Machine perfusion or cold storage in organ transplantation: indication, mechanisms, and future perspectives. Transplant international 23: 561-570.
73. Shoskes D, Lapierre C, Cruz-Correa M, Muruve N, Rosario R, et al. (2005) Beneficial effects of the bioflavonoids curcumin and quercetin on early function in cadaveric renal transplantation: a randomized placebo controlled trial Transplantation 80: 1556-1559.

74. Soares MP, Brouard S, Smith RN, Bach FH (2001) Heme oxygenase-1, protective gene that prevents the rejection of transplanted organs. Immuno Rev 184: 275-285.

75. Cendales LC, Kirk AD, Moresi JM, Ruiz P, Kleiner DE (2006) Composite tissue allotransplantation: classification of clinical acute skin rejection. Transplantation 81: 418-422.

This article was originally published in a special issue, Ischemia Reperfusion Injury handled by Editor(s). Dr. Manuel Maglione, Innsbruck Medical University, Austria; Dr. Pankaj Saxena, University of Western Australia, Australia 